QUARTERLY OF APPLIED MATHEMATICS

VOLUME LXIX, NUMBER 4

DECEMBER 2011, PAGES 723-746

S 0033-569X(2011)01245-6

Article electronically published on July 1, 2011

\title{
UNIFORM STABILIZATION OF A CLASS OF COUPLED SYSTEMS OF KDV EQUATIONS WITH LOCALIZED DAMPING
}

\author{
BY \\ C. P. MASSAROLO (Centro de Engenharias e Ciências Exatas, Universidade Estadual do Oeste do \\ Paraná, Av. Tarquínio Joslin dos Santos, 1300, CEP 85870-650, Foz do Iguaçu, PR, Brazil), \\ G. P. MENZALA (National Laboratory of Scientific Computation, LNCC/MCT, Rua Getulio Vargas, \\ 333, Quitandinha, Petrópolis, CEP 25651-070, RJ, Brasil and Institute of Mathematics, Federal \\ University of Rio de Janeiro, UFRJ, P.O. Box 68530, CEP 21945-970, Rio de Janeiro, RJ, Brasil), \\ AND \\ A. F. PAZOTO (Institute of Mathematics, Federal University of Rio de Janeiro, UFRJ, P.O. Box \\ 68530, CEP 21945-970, Rio de Janeiro, RJ, Brasil) \\ Abstract. We study the stabilization of solutions of a coupled system of Korteweg- \\ de Vries $(\mathrm{KdV})$ equations in a bounded interval under the effect of a localized damping \\ term. We use multiplier techniques combined with the so-called "compactness-uniqueness \\ argument". The problem is then reduced to proving a unique continuation property \\ (UCP) for weak solutions. The exponential decay of solutions was previously obtained in \\ Bisognin, Bisognin, and Menzala (2003) when the damping was effective simultaneously \\ in neighborhoods of both extremes of the bounded interval. In this work we address the \\ general case using a different approach to obtain the UCP and stabilize the system.
}

1. Introduction. This paper is devoted to studying the nonlinear coupled system of dispersive equations of the type

$$
U_{t}+U_{x}+D U_{x x x}+\lambda H(U) U_{x}+\mathcal{L}(x, B) U=0, \quad \text { in }(0, L) \times(0, \infty) .
$$

Received March 19, 2010.

2000 Mathematics Subject Classification. Primary 93D15, 93B05; Secondary 35B40, 35Q53.

Key words and phrases. Exponential decay, stabilization, Korteweg-de Vries equation.

CPM was supported by CNPq (Brazil) and MathAmsud.

GPM was supported by a Grant of CNPq, Proc. 301134/2009-0, PROSUL, Proc. 490329/2008-0 (Brazil)

and MathAmsud. He acknowledges very much such important support.

AFP was supported by CNPq, PROSUL and MathAmsud.

E-mail address: claiton@unioeste.br

E-mail address: perla@lncc.br

E-mail address: ademir@im.ufrj.br 
Here $U=\left(\begin{array}{l}u \\ v\end{array}\right)$, where $u$ and $v$ depend on $x \in(0, L)$ and time $t$,

$$
D=\left[\begin{array}{cc}
1 & a_{3} \\
a_{3} & 1
\end{array}\right], \text { with } 0<a_{3}<1, \quad H(U)=\left[\begin{array}{cc}
u+a_{2} v & a_{2} u+a_{1} v \\
a_{2} u+a_{1} v & a_{1} u+v
\end{array}\right]
$$

$a_{1}, a_{2} \in \mathbb{R}, \lambda>0$. $B$ is a linear bounded and positive operator, and $\mathcal{L}(x, B)$ is a linear bounded and positive operator which will be effective only on an open subset $\omega$ of the interval $(0, L)$. We will complement (1.1) with the boundary conditions

$$
U(0, t)=U(L, t)=U_{x}(L, t)=0, \quad \text { for any } t>0,
$$

and initial conditions

$$
U(x, 0)=U_{0}(x)=\left(\begin{array}{l}
u_{0}(x) \\
v_{0}(x)
\end{array}\right), 0<x<L .
$$

When $\mathcal{L} \equiv 0$, system (1.1) was derived by Gear and Grimshaw in [7] as a model to describe strong interactions of two long internal gravity waves in a stratified fluid, where the two waves are assumed to correspond to different modes of the linearized equations of motion. It has the structure of a pair of $\mathrm{KdV}$ equations with both linear and nonlinear coupling terms. This somewhat complicated system has been the object of intensive research in recent years (see, for instance, [2, [9] and [18]).

Our main result (see Theorem 1.1) says that the localized damping mechanism $\mathcal{L}(x, B)$ is enough in order to guarantee the exponential decay of the total energy as $t \rightarrow \infty$ as long as the initial data in (1.3) lives inside a ball of radius $R$ (no matter how large is $R>0)$. We will concentrate our attention on two important examples:

(I) Consider problem (1.1)-(1.3) with $B=I=$ identity and $\mathcal{L}(x, I) U=a(x) U$, where

$$
\left\{\begin{array}{l}
a \in L^{\infty}(0, L) \text { and } a(x) \geq a_{0}>0 \text { a.e. in } \omega, \\
\text { where } \omega \text { is a nonempty open subset of }(0, L) .
\end{array}\right.
$$

Therefore, the damping term is acting effectively in $\omega$. Let us consider the total energy associated to (1.1) in this case:

$$
E(t)=\frac{1}{2} \int_{0}^{L}\left(u^{2}+v^{2}\right) d x
$$

Using the boundary conditions, formally we can verify that

$$
\begin{aligned}
\frac{d E}{d t} & =-\int_{0}^{L} a(x)\left(u^{2}+v^{2}\right) d x-\frac{1}{2}\left[u_{x}^{2}(0, t)+v_{x}^{2}(0, t)+2 a_{3} u_{x}(0, t) v_{x}(0, t)\right] \\
& \leq-\int_{0}^{L} a(x)\left(u^{2}+v^{2}\right) d x-\frac{1}{2}\left(1-a_{3}\right)\left[u_{x}^{2}(0, t)+v_{x}^{2}(0, t)\right] \leq 0
\end{aligned}
$$

for any $t>0$, because $0<a_{3}<1$. Let us rewrite (1.6) as

$$
\frac{d E}{d t} \leq-a_{0}\|U\|_{\left[L^{2}(\omega)\right]^{2}}^{2}-C\left|U_{x}(0, t)\right|^{2}
$$

with $C=\frac{1}{2}\left(1-a_{3}\right)>0$. Thus, (1.7) indicates that $E(t)$ decreases along trajectories. 
(II) Consider $B=(-\Delta)^{-1}$ and $\mathcal{L}(x, B) U=1_{\omega}(-\Delta)^{-1} U=\left(\begin{array}{c}\left.(-\Delta)^{-1} u\right|_{\omega} \\ \left.(-\Delta)^{-1} v\right|_{\omega}\end{array}\right)$, where $1_{\omega}$ denotes the characteristic function of the set $\omega$ and $(-\Delta)^{-1}$ is the inverse of the operator $-\frac{d^{2}}{d x^{2}}$ in $\omega$ with Dirichlet boundary conditions (on the boundary of $\omega$ ).

Proceeding formally, we obtain the identity

$$
\begin{aligned}
& (U, \mathcal{L}(x, B) U)=\int_{\omega}(u B u+v B v) d x=-\int_{\omega}\left(\Delta^{-1} u\right) \Delta\left(\Delta^{-1} u\right) d x \\
& -\int_{\omega}\left(\Delta^{-1} v\right) \Delta\left(\Delta^{-1} v\right) d x+\left.\left\{(-\Delta)^{-1} u\left[(-\Delta)^{-1} u\right]_{x}+(-\Delta)^{-1} v\left[(-\Delta)^{-1} v\right]_{x}\right\}\right|_{\partial \omega} \\
& +\int_{\omega}\left(\left|\left[\Delta^{-1} u\right]_{x}\right|^{2}+\left|\left[\Delta^{-1} v\right]_{x}\right|^{2}\right) d x=\left\|\left[\Delta^{-1} u\right]_{x}\right\|_{L^{2}(\omega)}^{2}+\left\|\left[\Delta^{-1} v\right]_{x}\right\|_{L^{2}(\omega)}^{2} \\
& =\left\|\Delta^{-1} u\right\|_{H_{0}^{1}(\omega)}^{2}+\left\|\Delta^{-1} v\right\|_{H_{0}^{1}(\omega)}^{2}=\|U\|_{\left[H^{-1}(\omega)\right]^{2}}^{2} .
\end{aligned}
$$

Using the above identity and taking the inner product (in $X$ ) of (1.1) with $U=\left(\begin{array}{l}u \\ v\end{array}\right)$ we find

$$
\frac{d E}{d t} \leq-\|U\|_{\left[H^{-1}(\omega)\right]^{2}}^{2}-C\left|U_{x}(0, t)\right|^{2}
$$

where $E(t)=\frac{1}{2}\|U(\cdot, t)\|_{X}^{2}$ is the total energy associated to the model. Again, (1.9) indicates that $E(t)$ decreases along trajectories.

Due to (1.7) or (1.9) we observe that even when $a \equiv 0$ or $\omega=\emptyset$, the energy is dissipated through the extreme $x=0$. However, the dissipation due to the boundary terms $u_{x}(0, t)$ and $v_{x}(0, t)$ is not strong enough to guarantee the decay of solutions of (1.1) for all values of $L$. In fact, in [17, 18, it was proved that the decay of the solutions of the linearized system fail for some critical values of the length $L$ of the interval $(0, L)$.

Our main result reads as follows:

Theorem 1.1. Given any $R>0$, there exist $C=C(R)>0$ and $\mu=\mu(R)>0$ such that

$$
E(t) \leq C\left\|U_{0}\right\|_{\left[L^{2}(0, L)\right]^{2}}^{2} \exp (-\mu t)
$$

holds for all $t>0$ and any mild solution of (1.1) with $\left\|U_{0}\right\|_{X} \leq R$.

The arguments we shall describe in order to prove the (local) exponential decay of (1.1)-(1.3) are essentially the following:

i) We combine multiplier techniques developed in [22] for the analysis of controllability properties of the scalar KdV equation and the so-called "compactness-uniqueness argument" (see, for instance, [29]) in order to solve the following problem:

ii) To find $T>0$ and a positive constant $C$ such that

$$
E(0) \leq C\left\{\int_{0}^{T}\|U(\cdot, t)\|_{Y(\omega)}^{2} d t+\int_{0}^{T}\left|U_{x}(0, t)\right|^{2} d t\right\}
$$

holds for every finite energy solution of (1.1). Here $Y(\omega)$ may be a functional space somehow bigger than the usual $L^{2}(\omega) \times L^{2}(\omega)$.

The problem of obtaining (1.10) is reduced to showing that the unique solution of (1.1) such that $\mathcal{L}(x, B) U=0$ a.e. and $U_{x}(0, t)=0$ for any time $t$ has to be the trivial one. This problem may be viewed as a unique continuation property (UCP) for the solution 
$U$ of (1.1)-(1.3). If the steps $i$ ) and $i i)$ are solved, then using (1.10) together with the decreasing information of $E(t)$ it follows that there exists $0<\gamma<1$ and $T>0$ such that $E(T) \leq \gamma E(0)$, which, combined with the semigroup property, allows us to derive the (local) exponential decay of $E(t)$. We observe that for the examples mentioned above we will choose $Y(\omega)=\left[L^{2}(\omega)\right]^{2}$ and $\left[H^{-1}(\omega)\right]^{2}$ for the cases $i$ ) and $\left.i i\right)$, respectively.

The problem we address here has been intensively investigated in the context of wave equations but there are fewer results for the KdV type equation under the boundary condition as in (1.1). However, there are quite complete results in what concerns boundary controllability. Rosier in 22] proved that the underlying scalar linear equation without damping is exactly controllable by means of a single boundary control except when $L$ lies in a countable set of critical lengths of the form

$$
\mathcal{E}=\left\{\frac{2 \pi}{\sqrt{3}} \sqrt{k^{2}+k l+l^{2}}, k \text { and } l \text { are positive natural numbers }\right\} .
$$

This was done using multiplier techniques and Lions' HUM method (see [11]). The critical lengths in (1.11) are such that there are eigenfunctions of the linear scalar problem for which the observability inequality (1.10) leading to controllability fails when $a \equiv 0$. By a linearization argument, a local controllability result for the semilinear scalar equation was also proved in 22. Later on, Zhang in 28 proved that using three controls, acting on all the boundary conditions, controllability holds for all values of $L$. More recently, Crépeau and Coron [5] and Cerpa [3] proved that, for some critical values of the length $L$, the nonlinear scalar model is controllable (see also [4]).

When the damping function $a=a(x)$ is active simultaneously in a neighborhood of both extremes of the interval $(0, L)$ the problem was addressed in [16] for the scalar KdV equation

$$
u_{t}+u_{x}+u_{x x x}+u u_{x}+a(x) u=0, \quad \text { in }(0, L) \times(0, \infty)
$$

under boundary conditions as in (1.2). Following closely the multiplier techniques developed in 22 for the analysis of controllability properties, it was proved that the energy decays exponentially in bounded sets of initial data. However, when using multipliers, the nonlinearity produces extra terms which in [16] were handled by compactness-uniqueness. Thus, the problem is reduced to showing that the unique solution $u$ such that, $a(x) u=0$ everywhere and $u_{x}(0, t)=0$ for all time $t$, has to be the trivial one. This problem may be viewed as a unique continuation one since $a u=0$ implies that $u=0$ in $\{a>0\} \times(0, T)$. If $\omega$ contains a neighborhood of both extremes $x=0, L$, the result may be obtained in two steps, as in [16]: First, extending the solution by zero outside the interval $(0, L)$, we get a compactly supported (in space) solution of the Cauchy problem for the system of $\mathrm{KdV}$ equations on the whole line. Then, we apply the classical smoothing properties in 8] showing that the solution is smooth. This allows applying the unique continuation property results in [27] on smooth solutions to conclude that $u=0$.

The general case, that is, the case in which the function $a=a(x)$ is active in any open subset of $(0, L)$ was first solved in [19] for the scalar KdV equation (1.12) and boundary conditions as in (1.2). Combining the multiplier techniques developed in 22$]$ and the so-called "compactness-uniqueness arguments" (see, for instance, 29]) it was shown that the solutions vanishing on any subinterval of $(0, L)$ are necessarily smooth, 
which yielded enough regularity on $u$ to apply the unique continuation results obtained in 23] by Carleman inequalities. As a consequence, it was deduced that, for any $R>0$, there exist positive constants $C=C(R)$ and $\alpha=\alpha(R)$ such that

$$
\|u\|_{L^{2}(0, L)}^{2} \leq C(R)\left\|u_{0}\right\|_{L^{2}(0, L)}^{2} e^{-\alpha(R) t}, \quad t \geq 0
$$

provided $\left\|u_{0}\right\|_{L^{2}(0, L)}^{2} \leq R$. More recently, Rosier and Zhang [20] considered the generalized $\mathrm{KdV}$ model

$$
u_{t}+u_{x}+u_{x x x}+u^{p} u_{x}+a(x) u=0, \quad \text { in }(0, L) \times(0, \infty)
$$

with boundary conditions as in (1.2) and $p=2,3$. They established existence, uniqueness, and persistence properties of solutions corresponding to the given initial data $u_{0}$, together with continuous dependence of the solution upon the initial data $u_{0}$. Following the methods described above (multiplier techniques, compactness arguments and unique continuation property) the decay of solutions in the energy space was also obtained. At that point we observe that to obtain the decay of solutions the authors used a new unique continuation property whose proof is mainly based on a Carleman-type estimate for the Korteweg-de Vries equation established by Rosier in 21. The critical case $p=4$ was solved in 10 by considering data $u_{0}$ such that $\left\|u_{0}\right\|_{L^{2}(0, L)}$ is small.

In what concerns system (1.1), the problem of stabilization was first solved in 1] in the case $B=I$ and $\mathcal{L}(x, I) U=a(x) U$ when the damping function $a=a(x)$ is localized in a neighborhood of both extremes of the interval $(0, L)$. Performing as in [16, the authors combine the results of the smoothing property and unique continuation in [26] and [6], respectively, and derive the uniform exponential decay of the energy $E(t)$ provided $E(0) \leq R$, whenever we fix $R>0$.

The problem of stabilization for the scalar version of (1.1) in the case $B=\left(-\frac{d^{2}}{d x^{2}}\right)^{-1}$ and $\mathcal{L}(x, B) U=\left.\left(-\frac{d^{2}}{d x^{2}}\right)^{-1} u\right|_{\omega}$, where $\omega$ is a nonempty open subset of $(0, L)$, was considered in [14]. The corresponding techniques introduced by Pazoto in [19] were properly adapted in this case which could be thought of as a localized weak dissipation (in the $H^{-1}$-norm) of the scalar KdV.

In this article, inspired by the works [1, 17] and [19, we address the general case and show that the energy of the solutions of (1.1)-(1.3) decays exponentially as $t \rightarrow \infty$ when $\omega$ is any nonempty open subset of $(0, L)$. Arguing as in [19], the problem is reduced to showing that whenever $u \equiv v \equiv 0$ in $\omega \times(0, T)$ and $u_{x}(0, t)=v_{x}(0, t)=0$ for all time $t$ this yields enough regularity on $u$ and $v$ to apply the unique continuation results obtained in [6] and conclude that $u \equiv v \equiv 0$ in $(0, L) \times(0, T)$. We underscore that, due to the techniques we employ in this work, the unique continuation property is crucial to obtain the results of stabilization when the dissipation is localized in a subset of the domain where the equation holds. In this sense, the difficulty in studying system (1.1)(1.3) is introduced by the nonlinearity and the lack of smoothing effects to deal with it, since the unique continuation results may not be applied directly. For our purpose it will be enough to show the existence of a weak solution. Therefore, the intermediate step on the gain of regularity plays the central role in the proofs. Moreover, the result may be considered as a new contribution in the subject of proving unique continuation 
properties of weak solutions of partial differential equations, this time in the context of $\mathrm{KdV}$ equations.

Our result is of local nature in the sense that the exponential decay rate is uniform only in bounded sets of initial data, as in [16. However, the results obtained in this paper do not provide any estimate on how the decay rate depends on the radius $R$ of the ball. This has been done for nonlinear models, as far as we know, in very few cases and always using some structural conditions on the nonlinearity. We refer to 29 for the case of the semilinear wave equation with localized damping in which the uniform exponential decay is proved and to [15] for the analysis of the von Kármán system of thermoelastic plates where an explicit estimate on how the decay rate tends to zero as $R \rightarrow \infty$ is provided.

This paper is organized as follows. In Section 2, we present the main results of this work. Section 3 is devoted to proving our main result, i.e., the UCP of weak solutions of (1.1). In the Appendix, for the sake of completeness, we present the main steps to obtain the result of exponential decay of solutions.

2. Preliminary results. We consider problem (1.1)-(1.3) with $D, H(U)$ and $\lambda>0$ as in the introduction. In order to indicate briefly the existence and uniqueness of a global mild solution let us consider the space $X=L^{2}(\Omega) \times L^{2}(\Omega)$ and $B$ a linear bounded and positive operator with domain $D(B) \subseteq L^{2}(\Omega), B: D(B) \mapsto Z \subseteq L^{2}(\Omega)$ and $\mathcal{L}(x, B)$ : $D(\mathcal{L}) \subseteq X$ be a linear bounded operator $\mathcal{L}: D(\mathcal{L})=D(B) \times D(B) \mapsto A(x)[Z \times Z] \subseteq X$, where $A(x):(0, L) \rightarrow \mathbb{R}, A(x) \in L^{\infty}(0, L), A(x) \geq 0$ and there exists $A_{0}>0$ such that $A(x) \geq A_{0}>0$ a.e. in $\omega$, where $\omega$ is a nonempty subset of $(0, L)$.

Theorem 2.1. Let $U_{0} \in X$. Then, problem (1.1)-(1.3) has a unique global mild solution $U$ such that

$$
\|U\|_{\left.L^{\infty}(0, T ; X) \cap L^{2}\left(0, T ;\left[H_{0}^{1}(0, L)\right]^{2}\right)\right)} \leq C
$$

for any $T>0$, where $C$ is a positive constant which depends on $T$ and $\left\|U_{0}\right\|_{X}$.

Outline of the proof. We can proceed as in [1]. First we use semigroup theory to prove that the linear part of (1.1) is well-posed, i.e.,

$$
V_{t}+V_{x}+D V_{x x x}+\mathcal{L}(x, B) V=0, \quad(0, L) \times(0, \infty)
$$

with boundary and initial conditions of types (1.2) and (1.3). Then, local existence and uniqueness of (1.1)-(1.3) can be obtained using a fixed point argument. Global existence, uniqueness and (1.4) are obtained using the multiplier technique and the Sobolev embedding theorem.

The next theorem is crucial in order to obtain the main result of this paper.

Theorem 2.2. Let $U$ be the global mild solution of (1.1)-(1.3) obtained in Theorem 2.1. Suppose that

$$
U_{x}(0, t)=0 \text { for any } t \in(0, T)
$$

and

$$
U(x, t)=0 \text { for any }(x, t) \in \omega \times(0, T) \text {, a.e. in } x .
$$


Then, $U \in L^{2}\left(0, T ;\left[H^{3}(0, L)\right]^{2}\right) \cap H^{1}(0, T ; X)$.

REMARK 2.3. The above theorem gives us a gain of regularity of $U$ which is enough in order to use classical results to obtain the validity of the UCP in order to conclude that $U \equiv 0$.

Before proving Theorem 2.2, let us describe our strategy of proof in more detail. We use a similar approach to the one used in 19 where the scalar KdV equation was considered. First, we differentiate equation (1.1) with respect to the variable $t$ and analyze the regularity of $V=\left(\begin{array}{c}z \\ w\end{array}\right)=\left(\begin{array}{c}u_{t} \\ v_{t}\end{array}\right)$, which solves

$$
V_{t}+V_{x}+D V_{x x x}+\lambda H(V) U_{x}+\lambda H(U) V_{x}+\mathcal{L}(x, B) V=0, \quad \text { in }(0, L) \times(0, T)
$$

with boundary conditions

$$
V(0, t)=V(L, t)=V_{x}(L, t)=0, \quad t \in(0, T)
$$

and initial conditions

$$
V(x, 0)=V_{0}(x)=\left(\begin{array}{c}
z_{0}(x) \\
w_{0}(x)
\end{array}\right)=\left(\begin{array}{c}
u_{t}(x, 0) \\
v_{t}(x, 0)
\end{array}\right), \quad 0<x<L
$$

where $U=\left(\begin{array}{l}u \\ v\end{array}\right) \in L^{2}\left(0, T ;\left[H_{0}^{1}(0, L)\right]^{2}\right) \cap L^{\infty}(0, T ; X)$ is the weak solution of (1.1) and $V_{0} \in\left[H^{-3}(0, L)\right]^{2}$. Since $U=0$ in $\omega \times(0, T)$, then $V=0$ in $\omega \times(0, T)$ as well, $\omega$ being the subinterval where the damping $\mathcal{L}$ is effective. Thus, performing as in 19] (combining multiplier techniques and the so-called compactness-uniqueness argument) we show that the fact that $U=0$ in $\omega \times(0, T)$ implies the extra regularity property $V \in L^{2}\left(0, T ;\left[H_{0}^{1}(0, L)\right]^{2}\right) \cap L^{\infty}(0, T ; X)$ which yields enough regularity on $U$ to apply the UCP obtained in [6].

Note that the regularity result we prove on $(z, w)$ exhibits a definite gain of regularity since, in principle, the initial data of $V$ belongs to $\left[H^{-3}(0, L)\right]^{2}$. The gain of regularity comes from the fact that $V=0$ on $\omega \times(0, T)$.

In the sequel, we prove some technical results constituting the basic ingredients for obtaining the regularity result in Theorem 2.2.

Lemma 2.4. Let $U$ be the solution of problem (1.1)-(1.3) obtained in Theorem 2.1. Then, for any $V_{0} \in X$, problem (2.2)-(2.4) has a unique global mild solution $V \in$ $L^{2}\left(0, T ;\left[H_{0}^{1}(0, L)\right]^{2}\right) \cap L^{\infty}(0, T ; X)$ for any $T>0$.

Proof. The proof will be done in several steps, combining semigroup theory, a fixed point argument and energy estimates. Firstly, we will derive some estimates for the solutions of the underlying linear system

$$
\left\{\begin{array}{l}
V_{t}+V_{x}+D V_{x x x}+\mathcal{L}(x, B) V=0, \quad \text { in }(0, L) \times(0, T) \\
V(0, t)=V(L, t)=V_{x}(L, t)=0, \quad t \in(0, T) \\
V(x, 0)=V_{0}(x), \quad x \in(0, L) .
\end{array}\right.
$$

It is well known (see [1] and [6]) that, when $\mathcal{L} \equiv 0$, the operator

$$
A V=-\frac{\partial V}{\partial x}-D \frac{\partial^{3} V}{\partial x^{3}}
$$


with domain

$$
D(A)=\left\{U \in\left[H^{3}(0, L) \cap H_{0}^{1}(0, L)\right]^{2} ; U_{x}(L)=0\right\}
$$

generates a semigroup of contractions in $X$. Moreover, since $\mathcal{L}(x, B)$ is a bounded operator, then

$$
\|-\mathcal{L}(x, B) U\|_{X} \leq C\|U\|_{X},
$$

where $C$ is a positive constant. Then, from (2.6) we conclude that $A-\mathcal{L}(x, B)$ generates a semigroup of contractions $\{S(t)\}_{t \geq 0}$ in $X$. Clearly, from (2.5), using the energy method, we obtain

$$
\frac{d}{d t} \int_{0}^{L}|V|^{2} d x+\int_{0}^{L} V \cdot \mathcal{L}(x, B) V d x+\left|V_{x}(0, t)\right|^{2}=0
$$

In particular,

$$
\left\|S(t) V_{0}\right\|_{X} \leq\left\|V_{0}\right\|_{X}
$$

for any $t>0$. On the other hand, taking the inner product in $X$ of $(2.5)$ with $\left(\begin{array}{c}x z \\ x w\end{array}\right)$ and integrating over $(0, L) \times(0, T)$, we get

$$
\left\|S(.) V_{0}\right\|_{L^{2}\left(0, T ;\left[H_{0}^{1}(0, L)\right]^{2}\right)} \leq C(T)\left\|V_{0}\right\|_{X},
$$

where $C(T)$ is a positive constant given by

$$
C(T)=\sqrt{T+\frac{T+L}{3\left(1-a_{3}\right)}} .
$$

Now, in order to apply the fixed point argument, we consider the set

$$
\mathcal{K}(T)=L^{2}\left(0, T ;\left[H_{0}^{1}(0, L)\right]^{2}\right) \cap L^{\infty}(0, T ; X)
$$

endowed with the norm

$$
\|V\|_{\mathcal{K}(T)}=\|V\|_{L^{2}\left(0, T ;\left[H_{0}^{1}(0, L)\right]^{2}\right)}+\|V\|_{L^{\infty}(0, T ; X)} .
$$

Then, using the variation of parameters formula, system (2.2)-(2.4) may be written in the following integral form:

$$
V(t)=S(t) V_{0}+\int_{0}^{t} S(t-s) G(V(s)) d s:=P(V)(t),
$$

where $V=\left(\begin{array}{c}z \\ w\end{array}\right), U=\left(\begin{array}{c}u \\ v\end{array}\right)$ and $G$ is given by

$$
G(V)=-\lambda\left[H(V) U_{x}+H(U) V_{x}\right]=-\lambda\left(\begin{array}{c}
(u z)_{x}+a_{1}(v w)_{x}+a_{2}(v z+u w)_{x} \\
(v w)_{x}+a_{2}(u z)_{x}+a_{1}(v z+u w)_{x}
\end{array}\right) .
$$

In order to prove the local existence and uniqueness for system (2.2)-(2.4), we proceed in two steps:

Step 1: $P$ maps $\mathcal{K}(T)$ into itself continuously.

From (2.7) and (2.8) we deduce that

$$
\begin{aligned}
\left\|S(.) V_{0}\right\|_{\mathcal{K}(T)} & =\left\|S(.) V_{0}\right\|_{L^{\infty}(0, T ; X)}+\left\|S(.) V_{0}\right\|_{L^{2}\left(0, T ;\left[H_{0}^{1}(0, L)\right]^{2}\right)} \\
& \leq(1+C(T))\left\|V_{0}\right\|_{X},
\end{aligned}
$$


where $C(T)$ is given by (2.9). On the other hand, the function

$$
J(t)=\int_{0}^{t} S(t-s) G(V(s)) d s
$$

solves the system

$$
\left\{\begin{array}{l}
J_{t}+J_{x}+D J_{x x x}+\mathcal{L}(x, B) J=G(V), \quad \text { in }(0, L) \times(0, T) \\
J(0, t)=J(L, t)=J_{x}(L, t)=0, \quad t \in(0, T) \\
J(x, 0)=0, \quad x \in(0, L) .
\end{array}\right.
$$

But $G$ maps $\mathcal{K}(T) \subset L^{2}\left(0, T ;\left[H_{0}^{1}(0, L)\right]^{2}\right)$ into $L^{1}(0, T ; X)$ and $G$ is clearly continuous. Thus, proceeding as in Rosier [Proposition 4.1, 22]] (see also [1]), we can prove that the mild solution $J(t)$ of (2.15) belongs to $\mathcal{K}(T)$ and the map $G \mapsto J(t)$ which maps $L^{1}(0, T ; X)$ into $\mathcal{K}(T)$ is continuous. Consequently, $P$ maps $\mathcal{K}(T)$ into itself continuously. Step 2: $P$ is a contraction from a suitable ball $B_{R}$ of $\mathcal{K}(T)$ into itself when $T>0$ is small enough (both $R$ and $T$ depend on the size of the initial conditions $z_{0}, w_{0}$ in $X$ and the potentials $u, v$ in $\left.L^{2}\left(0, T ; H_{0}^{1}(0, L)\right) \cap L^{\infty}(0, T ; X)\right)$.

In fact, let $V=\left(\begin{array}{c}z \\ w\end{array}\right)$ and $W=\left(\begin{array}{c}z^{1} \\ w^{1}\end{array}\right)$ be elements of $\mathcal{K}(T)$. Then, from (2.7),

$$
\begin{aligned}
& \|P(V)-P(W)\|_{L^{\infty}(0, T ; X)} \\
& =\underset{0 \leq t \leq T}{\operatorname{ess} \sup _{0}}\left\|\int_{0}^{t} S(t-s)[G(V(s))-G(W(s))] d s\right\|_{X} \\
& \leq \quad \underset{0 \leq t \leq T}{\operatorname{ess} \sup _{0}} \int_{0}^{t}\|S(t-s)[G(V(s))-G(W(s))]\|_{X} d s \\
& \leq \underset{0 \leq t \leq T}{\operatorname{ess} \sup _{0}} \int_{0}^{t}\|G(V(s))-G(W(s))\|_{X} d s \\
& \leq \int_{0}^{T}\|G(V(s))-G(W(s))\|_{X} d s=\|G(V)-G(W)\|_{L^{1}(0, T ; X)} .
\end{aligned}
$$

Now, from (2.8),

$$
\begin{aligned}
& \|P(V)-P(W)\|_{L^{2}\left(0, T ;\left[H_{0}^{1}(0, L)\right]^{2}\right)} \\
& =\left\|\int_{0}^{t} S(t-s)[G(V(s))-G(W(s))] d s\right\|_{L^{2}\left(0, T ;\left[H_{0}^{1}(0, L)\right]^{2}\right)} \\
& \leq \int_{0}^{t}\|S(t-s)[G(V(s))-G(W(s))]\|_{L^{2}\left(0, T ;\left[H_{0}^{1}(0, L)\right]^{2}\right)} d s \\
& \leq \int_{0}^{t}\|C(t)[G(V(s))-G(W(s))]\|_{X} d s \leq C(T) \int_{0}^{T}\|G(V(s))-G(W(s))\|_{X} d s \\
& =C(T)\|G(V)-G(W)\|_{L^{1}(0, T ; X) .}
\end{aligned}
$$

Combining (2.16) and (2.17), we deduce that

$$
\|P(V)-P(W)\|_{\mathcal{K}(T)} \leq \max \{1, C(T)\}\|G(V)-G(W)\|_{L^{1}(0, T ; X)} .
$$

To estimate the right-hand side of (2.18) we proceed as follows. We define

$$
\alpha=\max \left\{1, \lambda,\left|a_{1}\right|,\left|a_{2}\right|\right\} .
$$


Then, applying triangular and Hölder inequalities we get

$$
\begin{aligned}
& \|G(V)-G(W)\|_{L^{1}(0, T ; X)}=\int_{0}^{T}\|G(V(s))-G(W(s))\|_{X} d s \\
& =\lambda \int_{0}^{T} \|(u z)_{x}+a_{1}(v w)_{x}+a_{2}(v z+u w)_{x}-\left(u z^{1}\right)_{x} \\
& -a_{1}\left(v w^{1}\right)_{x}-a_{2}\left(v z^{1}+u w^{1}\right)_{x} \|_{L^{2}(0, L)} d s \\
& +\lambda \int_{0}^{T} \|(v w)_{x}+a_{2}(u z)_{x}+a_{1}(v z+u w)_{x}-\left(v w^{1}\right)_{x} \\
& -a_{2}\left(u z^{1}\right)_{x}-a_{1}\left(v z^{1}+u w^{1}\right)_{x} \|_{L^{2}(0, L)} d s \\
& \leq 2 \alpha \int_{0}^{T}\left(\|u\|_{L^{\infty}(0, L)}+\|v\|_{L^{\infty}(0, L)}\right) \\
& \times\left(\left\|z_{x}-z_{x}^{1}\right\|_{L^{2}(0, L)}+\left\|w_{x}-w_{x}^{1}\right\|_{L^{2}(0, L)}\right) d s \\
& +2 \alpha \int_{0}^{T}\left(\left\|z-z^{1}\right\|_{L^{\infty}(0, L)}+\left\|w-w^{1}\right\|_{L^{\infty}(0, L)}\right) \\
& \times\left(\left\|u_{x}\right\|_{L^{2}(0, L)}+\left\|v_{x}\right\|_{L^{2}(0, L)}\right) d s \\
& \leq 4 \alpha\left(\int_{0}^{T}\|u\|_{L^{\infty}(0, L)}^{2}+\|v\|_{L^{\infty}(0, L)}^{2} d s\right)^{\frac{1}{2}} \\
& \times\left(\int_{0}^{T}\left\|z_{x}-z_{x}^{1}\right\|_{L^{2}(0, L)}^{2}+\left\|w_{x}-w_{x}^{1}\right\|_{L^{2}(0, L)}^{2} d s\right)^{\frac{1}{2}} \\
& +4 \alpha\left(\int_{0}^{T}\left\|z-z^{1}\right\|_{L^{\infty}(0, L)}^{2}+\left\|w-w^{1}\right\|_{L^{\infty}(0, L)}^{2} d s\right)^{\frac{1}{2}} \\
& \times\left(\int_{0}^{T}\left\|u_{x}\right\|_{L^{2}(0, L)}^{2}+\left\|v_{x}\right\|_{L^{2}(0, L)}^{2} d s\right)^{\frac{1}{2}} \\
& \leq 4 \alpha\left\{\|U\|_{L^{2}\left(0, T ;\left[L^{\infty}(0, L)\right]^{2}\right)}\|V-W\|_{\mathcal{K}(T)}\right. \\
& \left.+\|V-W\|_{L^{2}\left(0, T ;\left[L^{\infty}(0, L)\right]^{2}\right)}\|U\|_{\mathcal{K}(T)}\right\} .
\end{aligned}
$$

Now, we use the classical interpolation inequality (Gagliardo-Nirenberg): There exists $\gamma>0$ such that

$$
\|h\|_{L^{\infty}(0, L)} \leq \gamma\|h\|_{L^{2}(0, L)}^{\frac{1}{2}}\left\|h_{x}\right\|_{L^{2}(0, L)}^{\frac{1}{2}}, \quad \forall h \in H_{0}^{1}(0, L) .
$$


As a consequence, we have

$$
\begin{aligned}
& \|U\|^{2} \\
& \quad L^{2}\left(0, T ;\left[L^{\infty}(0, L)\right]^{2}\right)=\int_{0}^{T}\left(\|u\|_{L^{\infty}(0, L)}^{2}+\|v\|_{L^{\infty}(0, L)}^{2}\right) d s \\
& \leq \gamma^{2} \int_{0}^{T}\left(\|u\|_{L^{2}(0, L)}\left\|u_{x}\right\|_{L^{2}(0, L)}+\|v\|_{L^{2}(0, L)}\left\|v_{x}\right\|_{L^{2}(0, L)}\right) d s \\
& \leq \gamma^{2}\left(\int_{0}^{T}\|u\|_{L^{2}(0, L)}^{2} d s\right)^{\frac{1}{2}}\left(\int_{0}^{T}\left\|u_{x}\right\|_{L^{2}(0, L)}^{2} d s\right)^{\frac{1}{2}} \\
& \quad+\gamma^{2}\left(\int_{0}^{T}\|v\|_{L^{2}(0, L)}^{2} d s\right)^{\frac{1}{2}}\left(\int_{0}^{T}\left\|v_{x}\right\|_{L^{2}(0, L)}^{2} d s\right)^{\frac{1}{2}} \\
& \leq \gamma^{2} T^{\frac{1}{2}}\left\{\|u\|_{L^{\infty}\left(0, T ; L^{2}(0, L)\right)}\|u\|_{L^{2}\left(0, T ; H_{0}^{1}(0, L)\right)}\right. \\
& \left.\quad+\|v\|_{L^{\infty}\left(0, T ; L^{2}(0, L)\right)}\|v\|_{L^{2}\left(0, T ; H_{0}^{1}(0, L)\right)}\right\} \\
& \leq \gamma^{2} T^{\frac{1}{2}}\|U\|_{L^{\infty}(0, T ; X)}\|U\|_{L^{2}\left(0, T ;\left[H_{0}^{1}(0, L)\right]^{2}\right)} \leq \gamma^{2} T^{\frac{1}{2}}\|U\|_{\mathcal{K}(T)}^{2},
\end{aligned}
$$

i.e.,

$$
\|U\|_{L^{2}\left(0, T ;\left[L^{\infty}(0, L)\right]^{2}\right)} \leq \gamma T^{\frac{1}{4}}\|U\|_{\mathcal{K}(T)} .
$$

Analogously, we obtain

$$
\|V-W\|_{L^{2}\left(0, T ;\left[L^{\infty}(0, L)\right]^{2}\right)} \leq \gamma T^{\frac{1}{4}}\|V-W\|_{\mathcal{K}(T)} .
$$

Now, returning to (2.20) and taking (2.23) and (2.24) into account, we deduce that

$$
\|G(V)-G(W)\|_{L^{1}(0, T ; X)} \leq 8 \alpha \gamma T^{\frac{1}{4}}\|U\|_{\mathcal{K}(T)}\|V-W\|_{\mathcal{K}(T)} .
$$

Finally, combining (2.25) and (2.18), the following holds:

$$
\|P(V)-P(W)\|_{\mathcal{K}(T)} \leq 8 \max \{1, C(T)\} \alpha \gamma T^{\frac{1}{4}}\|U\|_{\mathcal{K}(T)}\|V-W\|_{\mathcal{K}(T)} .
$$

Inequality (2.26) allows us to conclude that $P$ is a contraction in the ball $B_{R}=\{V \in$ $\left.\mathcal{K}(T):\|V\|_{\mathcal{K}(T)} \leq R\right\}$ whenever

$$
8 \max \left\{1, \sqrt{T+\frac{T+L}{3\left(1-a_{3}\right)}}\right\} \alpha \gamma T^{\frac{1}{4}}\|U\|_{\mathcal{K}(T)}<1,
$$

which will be true as long as $T>0$ is chosen sufficiently small.

In view of the computations above, the proof of Step 2 will be complete if we show that $P$ maps $B_{R}$ into itself for a suitable choice of $R$ and $T$ satisfying (2.27). Indeed, for all $V \in B_{R}$, estimative (2.26) gives us that

$$
\|P(V)\|_{\mathcal{K}(T)} \leq\|P(0)\|_{\mathcal{K}(T)}+\|P(V)-P(0)\|_{\mathcal{K}(T)} .
$$

Next, taking $W=0$ in (2.26) and using (2.7) and (2.8) we obtain

$$
\begin{aligned}
\|P(V)\|_{\mathcal{K}(T)} & \leq\left\|S(.) V_{0}\right\|_{\mathcal{K}(T)}+8 \max \{1, C(T)\} \alpha T^{\frac{1}{4}} \gamma\|U\|_{\mathcal{K}(T)} R \\
& \leq\left\|V_{0}\right\|_{X}+C(T)\left\|V_{0}\right\|_{X}+8 \max \{1, C(T)\} \alpha T^{\frac{1}{4}} \gamma\|U\|_{\mathcal{K}(T)} R \\
& =(1+C(T))\left\|V_{0}\right\|_{X}+8 \max \{1, C(T)\} \alpha T^{\frac{1}{4}} \gamma\|U\|_{\mathcal{K}(T)} R .
\end{aligned}
$$


Thus, choosing $R=\left(1+\sqrt{1+\frac{1+L}{1-a_{3}}}\right)\left\|V_{0}\right\|_{X}$ we deduce that

$$
\begin{aligned}
& \|P(V)\|_{\mathcal{K}(T)} \\
& \leq\left\{1+C(T)+8 \max \{1, C(T)\} \alpha T^{\frac{1}{4}} \gamma\|U\|_{\mathcal{K}(T)}\left(1+\sqrt{1+\frac{1+L}{1-a_{3}}}\right)\right\}\left\|V_{0}\right\|_{X} .
\end{aligned}
$$

In order to guarantee that the right-hand side of (2.29) is less than $R$, we choose $T>0$, sufficiently small, such that

$$
C(T)+8 \max \{1, C(T)\} \alpha T^{\frac{1}{4}} \gamma\|U\|_{\mathcal{K}(T)}\left(1+\sqrt{1+\frac{1+L}{1-a_{3}}}\right)<\left(\sqrt{1+\frac{1+L}{1-a_{3}}}\right),
$$

where $C(T)$ is given by (2.9). This is always possible. Taking $T>0$ possibly smaller allows us to guarantee (2.27) as well. This concludes the proof of Step 2.

This shows that system (2.2)-(2.4) has a unique mild solution for $0 \leq t<T$, with $T$ small. Thus, in order to conclude the proof of Lemma 2.4, it is sufficient to prove that this solution exists globally. To do that we need some a priori estimates, which will be obtained in several steps.

Step 3: This step is devoted to obtaining estimates for $V=\left(\begin{array}{c}z \\ w\end{array}\right)$ in $X$. More precisely, we will show that

$$
\int_{0}^{L}\|V(\cdot, T)\|^{2} d x \leq \int_{0}^{L}\left\|V_{0}\right\|^{2} d x+2 \alpha \int_{0}^{T}\|U\|_{\left[L^{\infty}(0, L)\right]^{2}}^{2}\|V\|_{X}^{2} d t+4 \alpha \int_{0}^{T}\left\|V_{x}\right\|_{X}^{2} d t
$$

for $\alpha$ defined in (2.19).

We take the inner product in $X$ of (2.2) with $V=\left(\begin{array}{c}z \\ w\end{array}\right)$ and integrate by parts over $(0, L)$ to obtain

$$
\begin{aligned}
& \frac{1}{2} \frac{d}{d t} \int_{0}^{L}\left(z^{2}+w^{2}\right) d x \\
& +\underbrace{\frac{1}{2}\left[z_{x}^{2}(0, t)+w_{x}^{2}(0, t)\right]+a_{3} z_{x}(0, t) w_{x}(0, t)+(\mathcal{L}(x, B) U, U)}_{\geq 0} \\
& =\int_{0}^{L} u z z_{x} d x+\int_{0}^{L} v w w_{x} d x+a_{1} \int_{0}^{L} v w z_{x} d x+a_{2} \int_{0}^{L} u z w_{x} d x \\
& +a_{2} \int_{0}^{L} v z z_{x} d x+a_{2} \int_{0}^{L} u w z_{x} d x+a_{1} \int_{0}^{L} v z w_{x} d x+a_{1} \int_{0}^{L} u w w_{x} d x
\end{aligned}
$$


or

$$
\begin{aligned}
\frac{1}{2} \int_{0}^{L}\left[z^{2}(x, T)+w^{2}(x, T)\right] d x & -\frac{1}{2} \int_{0}^{L}\left(z_{0}^{2}+w_{0}^{2}\right) d x \\
\leq & \alpha[\underbrace{\int_{0}^{T} \int_{0}^{L}\left|u z z_{x}\right| d x d t}_{i}+\underbrace{\int_{0}^{T} \int_{0}^{L}\left|v w w_{x}\right| d x d t}_{i i}+\underbrace{\int_{0}^{T} \int_{0}^{L}\left|v w z_{x}\right| d x d t}_{i i i} \\
& +\underbrace{\int_{0}^{T} \int_{0}^{L}\left|u z w_{x}\right| d x d t}_{v i i}+\underbrace{\int_{0}^{T} \int_{0}^{L}\left|v z z_{x}\right| d x d t}_{v i i i}+\underbrace{\int_{0}^{T} \int_{0}^{L}\left|u w z_{x}\right| d x d t}_{0} \\
& +\underbrace{\int_{0}^{T} \int_{0}^{T} \int_{0}^{L}\left|u w w_{x}\right| d x d t}_{\int_{0}^{L}\left|v z w_{x}\right| d x d t}] .
\end{aligned}
$$

Now, applying the Young and Hölder inequalities in the first term of (2.32) we get

$$
\begin{aligned}
\int_{0}^{T} \int_{0}^{L}\left|u z z_{x}\right| d x d t & \leq \int_{0}^{T}\|u z\|_{L^{2}(0, L)}\left\|z_{x}\right\|_{L^{2}(0, L)} d t \\
& \leq \frac{1}{2} \int_{0}^{T}\|u z\|_{L^{2}(0, L)}^{2} d t+\frac{1}{2} \int_{0}^{T}\left\|z_{x}\right\|_{L^{2}(0, L)}^{2} d t \\
& \leq \frac{1}{2} \int_{0}^{T}\|u\|_{L^{\infty}(0, L)}^{2}\|z\|_{L^{2}(0, L)}^{2} d t+\frac{1}{2} \int_{0}^{T}\left\|z_{x}\right\|_{L^{2}(0, L)}^{2} d t
\end{aligned}
$$

Performing similar calculations to those leading to (2.33) we can estimate the terms $(i i)$ up to (viii) in the right of (2.32) to obtain

$$
\begin{aligned}
& \int_{0}^{L}\left[z^{2}(x, T)+w^{2}(x, T)\right] d x \leq \int_{0}^{L}\left(z_{0}^{2}+w_{0}^{2}\right) d x \\
& +2 \alpha \int_{0}^{T}\left(\|u\|_{L^{\infty}(0, L)}^{2}+\|v\|_{L^{\infty}(0, L)}^{2}\right)\left(\|z\|_{L^{2}(0, L)}^{2}+\|w\|_{L^{2}(0, L)}^{2}\right) d t \\
& +4 \alpha \int_{0}^{T}\left(\left\|z_{x}\right\|_{L^{2}(0, L)}^{2}+\left\|w_{x}\right\|_{L^{2}(0, L)}^{2}\right) d t .
\end{aligned}
$$

Step 4: Now, we will estimate the last term in (2.34). We claim that the following holds:

$$
\begin{aligned}
& \int_{0}^{T} \int_{0}^{L}\left(z_{x}^{2}+w_{x}^{2}\right) d x d t \leq \frac{C}{\delta}\left[\int_{0}^{L}\left(z_{0}^{2}+w_{0}^{2}\right) d x\right. \\
& \left.+\int_{0}^{T}\left(1+\|u\|_{L^{\infty}(0, L)}^{2}+\|v\|_{L^{\infty}(0, L)}^{2}\right)\left(\|z\|_{L^{2}(0, L)}^{2}+\|w\|_{L^{2}(0, L)}^{2}\right) d t\right]
\end{aligned}
$$

for some constants $C>0$ and $\delta>0$ sufficiently small.

In order to prove the claim, we take the inner product in $X$ of (2.2) with $\left(\begin{array}{c}x z \\ x w\end{array}\right)$ and integrate over $(0, L) \times(0, T)$. Then, performing integration by parts and using the 
boundary conditions we obtain the following identity:

$$
\begin{aligned}
\int_{0}^{T} \int_{0}^{L}\left(z_{x}^{2}+w_{x}^{2}\right) d x d t \\
+\underbrace{\frac{1}{3} \int_{0}^{L} x\left[z^{2}(x, T)+w^{2}(x, T)\right] d x+\frac{2}{3} \int_{0}^{T}(\mathcal{L}(x, B) V, V) d t}_{\geq 0} \\
=-2 a_{3} \int_{0}^{T} \int_{0}^{L} z_{x} w_{x} d x d t+\frac{1}{3} \int_{0}^{T} \int_{0}^{L}\left(z^{2}+w^{2}\right) d x d t \\
+\frac{1}{3} \int_{0}^{L} x\left(z_{0}^{2}+w_{0}^{2}\right) d x+\frac{2}{3} \int_{0}^{T} \int_{0}^{L} x u z z_{x} d x d t+\frac{2}{3} \int_{0}^{T} \int_{0}^{L} u z^{2} d x d t \\
+\frac{2}{3} \int_{0}^{T} \int_{0}^{L} v w^{2} d x d t+\frac{4}{3} a_{1} \int_{0}^{T} \int_{0}^{L} v z w d x d t+\frac{4}{3} a_{2} \int_{0}^{T} \int_{0}^{L} u z w d x d t \\
+\frac{2}{3} a_{1} \int_{0}^{T} \int_{0}^{L} x v z w_{x} d x d t+\frac{2}{3} a_{1} \int_{0}^{T} \int_{0}^{L} x v z_{x} w d x d t \\
+\frac{2}{3} a_{2} \int_{0}^{T} \int_{0}^{L} x u z_{x} w d x d t+\frac{2}{3} a_{2} \int_{0}^{T} \int_{0}^{L} x u z w_{x} d x d t+\frac{2}{3} a_{1} \int_{0}^{T} \int_{0}^{L} u w^{2} d x d t \\
+\frac{2}{3} a_{2} \int_{0}^{T} \int_{0}^{L} v z^{2} d x d t+\frac{2}{3} a_{1} \int_{0}^{T} \int_{0}^{L} x u w w_{x} d x d t \\
+\frac{2}{3} a_{2} \int_{0}^{T} \int_{0}^{L} x v z z_{x} w d x d t+\frac{2}{3} \int_{0}^{T} \int_{0}^{L} x v w w_{x} d x d t .
\end{aligned}
$$

Since $2 a_{3} \int_{0}^{T} \int_{0}^{L} z_{x} w_{x} d x d t \geq-a_{3} \int_{0}^{T} \int_{0}^{L}\left(z_{x}^{2}+w_{x}^{2}\right) d x d t$ and the terms in the brace are nonnegative as indicated, from (2.36) it follows that

$$
\begin{aligned}
& \int_{0}^{T} \int_{0}^{L}\left(z_{x}^{2}+w_{x}^{2}\right) d x d t \leq \beta\left[\int_{0}^{T} \int_{0}^{L}\left(z^{2}+w^{2}\right) d x d t+\int_{0}^{L}\left(z_{0}^{2}+w_{0}^{2}\right) d x\right. \\
& +\underbrace{\int_{0}^{T} \int_{0}^{L}\left|u z z_{x}\right| d x d t}_{i}+\underbrace{\int_{0}^{T} \int_{0}^{L}\left|v w w_{x}\right| d x d t}_{i i}+\underbrace{\int_{0}^{T} \int_{0}^{L}\left|v w z_{x}\right| d x d t}_{i i i} \\
& +\underbrace{\int_{0}^{T} \int_{0}^{L}\left|u z w_{x}\right| d x d t}_{i v}+\underbrace{\int_{0}^{T} \int_{0}^{L}\left|v z z_{x}\right| d x d t}_{v}+\underbrace{\int_{0}^{T} \int_{0}^{L}\left|u w z_{x}\right| d x d t}_{v i} \\
& +\underbrace{\int_{0}^{T} \int_{0}^{L}\left|v z w_{x}\right| d x d t}_{v i i}+\underbrace{\int_{0}^{T} \int_{0}^{L}\left|u w w_{x}\right| d x d t}_{v i i i}+\underbrace{\int_{0}^{T} \int_{0}^{L^{v i}} u z^{2} d x d t}_{i x} \\
& +\underbrace{\int_{0}^{T} \int_{0}^{L} v w^{2} d x d t}_{x}+\underbrace{\int_{0}^{T} \int_{0}^{L} u w^{2} d x d t}_{x i}+\underbrace{\int_{0}^{T} \int_{0}^{L} v z^{2} d x d t}_{x i i}],
\end{aligned}
$$


where

$$
\beta=\frac{1}{1-a_{3}} \max \left\{\frac{2}{3}, \frac{2}{3} L, \frac{4}{3}\left|a_{1}\right|, \frac{4}{3}\left|a_{2}\right|, \frac{2}{3} L\left|a_{1}\right|, \frac{2}{3} L\left|a_{2}\right|\right\} .
$$

Performing as in (2.33) we can estimate the term $(i)$ as follows: For any $\delta_{1}>0$ we have

$$
\begin{aligned}
& \int_{0}^{T} \int_{0}^{L}\left|u z z_{x}\right| d x d t \leq\left(\int_{0}^{T} \frac{1}{\delta_{1}}\|u z\|_{L^{2}(0, L)}^{2} d t\right)^{\frac{1}{2}}\left(\int_{0}^{T} \delta_{1}\left\|z_{x}\right\|_{L^{2}(0, L)}^{2} d t\right)^{\frac{1}{2}} \\
& \leq \frac{1}{2 \delta_{1}} \int_{0}^{T}\|u\|_{L^{\infty}(0, L)}^{2}\|z\|_{L^{2}(0, L)}^{2} d t+\frac{\delta_{1}}{2} \int_{0}^{T}\left\|z_{x}\right\|_{L^{2}(0, L)}^{2} d t .
\end{aligned}
$$

In a similar way we can estimate the terms (ii) up to (viii) in (2.37). Moreover, applying Poincaré's inequality in $(i x)$, we deduce that for any $\delta_{2}>0$ we have

$$
\begin{aligned}
& \int_{0}^{T} \int_{0}^{L}\left|u z^{2}\right| d x d t \leq \int_{0}^{T}\|u z\|_{L^{2}(0, L)}\|z\|_{L^{2}(0, L)} d t \\
& \leq \frac{1}{2 \delta_{2}} \int_{0}^{T}\|u\|_{L^{\infty}(0, L)}^{2}\|z\|_{L^{2}(0, L)}^{2} d t+\frac{\delta_{2}}{2} C_{p}^{2} \int_{0}^{T}\left\|z_{x}\right\|_{L^{2}(0, L)}^{2} d t,
\end{aligned}
$$

where $C_{p}$ denotes Poincaré's constant. Analogously, we estimate the terms $(x)$ up to (xii) in the right-hand side of (2.37).

Finally, combining (2.37), (2.39) and (2.40) we obtain

$$
\begin{aligned}
& \int_{0}^{T} \int_{0}^{L}\left(z_{x}^{2}+w_{x}^{2}\right) d x d t \leq \frac{C}{\delta}\left[\int_{0}^{L}\left(z_{0}^{2}+w_{0}^{2}\right) d x\right. \\
& \left.+\int_{0}^{T}\left(1+\|u\|_{L^{\infty}(0, L)}^{2}+\|v\|_{L^{\infty}(0, L)}^{2}\right)\left(\|z\|_{L^{2}(0, L)}^{2}+\|w\|_{L^{2}(0, L)}^{2}\right) d t\right]
\end{aligned}
$$

for some constants $C>0$ and $\delta>0$ sufficiently small. This completes Step 4 .

Now, we can conclude the proof of Lemma 2.4 i.e., the global well-posedness for system (2.2)-(2.4). Replacing (2.35) into (2.34) we have

$$
\begin{aligned}
& \int_{0}^{L}\left[z^{2}(x, T)+w^{2}(x, T)\right] d x \leq C\left[\int_{0}^{L}\left(z_{0}^{2}+w_{0}^{2}\right) d x\right. \\
& \left.+\int_{0}^{T}\left(1+\|u\|_{L^{\infty}(0, L)}^{2}+\|v\|_{L^{\infty}(0, L)}^{2}\right)\left(\|z\|_{L^{2}(0, L)}^{2}+\|w\|_{L^{2}(0, L)}^{2}\right) d t\right]
\end{aligned}
$$

for some positive constant $C>0$. Then, applying Gronwall's inequality together with Theorem 2.1, we deduce that

$$
\|(z, w)\|_{L^{\infty}(0, T ; X)}=\underset{0 \leq t \leq T}{\operatorname{ess} \sup }\|(z(\cdot, t), w(\cdot, t))\|_{X} \leq C,
$$

where $C=C\left(T, \beta,\left\|u_{0}\right\|_{L^{2}(0, L)},\left\|v_{0}\right\|_{L^{2}(0, L)},\left\|z_{0}\right\|_{L^{2}(0, L)},\left\|w_{0}\right\|_{L^{2}(0, L)}\right)>0$.

On the other hand, combining (2.35), (2.42) and Theorem 2.1, we obtain

$$
\|(z, w)\|_{L^{2}\left(0, T ;\left[H_{0}^{1}(0, L)\right]^{2}\right)}^{2} \leq C,
$$

where $C>0$ also depends on $\left\|u_{0}\right\|_{L^{2}(0, L)},\left\|v_{0}\right\|_{L^{2}(0, L)},\left\|z_{0}\right\|_{L^{2}(0, L)},\left\|w_{0}\right\|_{L^{2}(0, L)}, \beta$ and $T$. This concludes the proof of Lemma 2.4. 
Lemma 2.5. Given $R$, there exists a positive constant $C=C\left(T, L,\left\|u_{0}\right\|_{L^{2}(0, L)}\right.$, $\left.\left\|v_{0}\right\|_{L^{2}(0, L)}, \alpha\right)$ such that

$$
\left\|V_{0}\right\|_{X}^{2} \leq C\left\{\int_{0}^{T}\left|V_{x}(0, t)\right|^{2} d t+\int_{0}^{T}\|V\|_{Y(\omega)}^{2} d t+\left\|V_{0}\right\|_{\left[H^{-3}(0, L)\right]^{2}}^{2}\right\}
$$

holds for every solution $V$ of (2.2)-(2.4) obtained in Lemma2.4 whose initial data satisfies $\left\|V_{0}\right\|_{X} \leq R$.

Proof. To prove (2.44) we combine multiplier techniques and the so-called "compactness-uniqueness" argument. We first take the inner product in $X$ of $(2.2)$ with $\left(\begin{array}{c}(T-t) z \\ (T-t) w\end{array}\right)$. Next, performing integration by parts over $(0, L) \times(0, T)$, we obtain the following identity:

$$
\begin{aligned}
T \int_{0}^{L}\left(z_{0}^{2}+w_{0}^{2}\right) d x= & \int_{0}^{T} \int_{0}^{L}\left(z^{2}+w^{2}\right) d x d t+\int_{0}^{T}(T-t)\left(z_{x}^{2}(0, t)+w_{x}^{2}(0, t)\right) d t \\
& +2 a_{3} \int_{0}^{T}(T-t) z_{x}(0, t) w_{x}(0, t) d t+2 \int_{0}^{T}(T-t)(\mathcal{L}(x, B) V, V) d t \\
& +\int_{0}^{T} \int_{0}^{L}(T-t) u_{x} z^{2} d x d t+\int_{0}^{T} \int_{0}^{L}(T-t) v_{x} w^{2} d x d t \\
& +a_{2} \int_{0}^{T} \int_{0}^{L}(T-t) v_{x} z^{2} d x d t+a_{1} \int_{0}^{T} \int_{0}^{L}(T-t) u_{x} w^{2} d x d t \\
& +a_{2} \int_{0}^{T} \int_{0}^{L}(T-t) u_{x} z w d x d t+a_{1} \int_{0}^{T} \int_{0}^{L}(T-t) v_{x} z w d x d t .
\end{aligned}
$$

From (2.45) and the assumptions on $\mathcal{L}(x, B)$ we deduce that

$$
\begin{aligned}
& \int_{0}^{L}\left(z_{0}^{2}+w_{0}^{2}\right) d x \leq \frac{1}{T} \int_{0}^{T} \int_{0}^{L}\left(z^{2}+w^{2}\right) d x d t+2 \int_{0}^{T}\|V\|_{\left[L^{2}(\omega)\right]^{2}}^{2} d t \\
& +\left(1+a_{3}\right) \int_{0}^{T}\left(z_{x}^{2}(0, t)+w_{x}^{2}(0, t)\right) d t+2 \alpha \int_{0}^{T} \int_{0}^{L}\left(\left|u_{x}\right|+\left|v_{x}\right|\right)\left(z^{2}+w^{2}\right) d x d t .
\end{aligned}
$$

In the sequel, we estimate the terms on the right-hand side of (2.46). Applying Hölder's inequality, we get

$$
\begin{aligned}
\int_{0}^{T} \int_{0}^{L}\left|u_{x}\right| z^{2} d x d t & \leq \int_{0}^{T}\left\|u_{x}\right\|_{L^{2}(0, L)}\|z\|_{L^{4}(0, L)}^{2} d t \\
& \leq\left(\int_{0}^{T}\left\|u_{x}\right\|_{L^{2}(0, L)}^{2} d t\right)^{\frac{1}{2}}\left(\int_{0}^{T}\|z\|_{L^{4}(0, L)}^{4} d t\right)^{\frac{1}{2}} \\
& =\|u\|_{L^{2}\left(0, T ; H_{0}^{1}(0, L)\right)}\|z\|_{L^{4}\left(0, T ; L^{4}(0, L)\right)}^{2} .
\end{aligned}
$$

Similar arguments imply that the last integral in (2.46) satisfies

$$
\begin{aligned}
\int_{0}^{T} \int_{0}^{L}\left(\left|u_{x}\right|+\left|v_{x}\right|\right)\left(z^{2}+w^{2}\right) d x d t \leq & \left(\|u\|_{L^{2}\left(0, T ; H_{0}^{1}(0, L)\right)}+\|v\|_{L^{2}\left(0, T ; H_{0}^{1}(0, L)\right)}\right) \\
& \times\left(\|z\|_{L^{4}\left(0, T ; L^{4}(0, L)\right)}^{2}+\|w\|_{L^{4}\left(0, T ; L^{4}(0, L)\right)}^{2}\right) .
\end{aligned}
$$


Again, applying Hölder's inequality we get

$$
\begin{aligned}
& \frac{1}{T} \int_{0}^{T} \int_{0}^{L} z^{2} d x d t \leq \frac{\sqrt{L}}{T} \int_{0}^{T}\|z\|_{L^{4}(0, L)}^{2} d t \\
& \leq \frac{\sqrt{L}}{T}\left(\int_{0}^{T} 1^{2} d t\right)^{\frac{1}{2}}\left(\int_{0}^{T}\|z\|_{L^{4}(0, L)}^{4} d t\right)^{\frac{1}{2}} \\
& =\sqrt{\frac{L}{T}}\|z\|_{L^{4}\left(0, T ; L^{4}(0, L)\right)}^{2} .
\end{aligned}
$$

Now, returning to (2.46) and using the above estimates together with Theorem 2.1 we obtain

$$
\begin{aligned}
& \int_{0}^{T}\left(z_{0}^{2}+w_{0}^{2}\right) d t \leq c\left(\|z\|_{L^{4}\left(0, T ; L^{4}(0, L)\right)}^{2}+\|w\|_{L^{4}\left(0, T ; L^{4}(0, L)\right)}^{2}\right) \\
& +\left(1+a_{3}\right) \int_{0}^{T}\left(z_{x}^{2}(0, t)+w_{x}^{2}(0, t)\right) d t+2 \int_{0}^{T}\|V\|_{\left[L^{2}(\omega)\right]^{2}}^{2} d t,
\end{aligned}
$$

where $c>0$ depends only on $T, L,\left\|u_{0}\right\|_{L^{2}(0, L)},\left\|v_{0}\right\|_{L^{2}(0, L)}$ and $\alpha$. Thus, in order to prove (2.44) it is sufficient to show that for any $T>0$ there exists a positive constant $C=C(T)$ such that

$$
\begin{aligned}
\|z\|_{L^{4}\left(0, T ; L^{4}(0, L)\right)}^{2}+\|w\|_{L^{4}\left(0, T ; L^{4}(0, L)\right)}^{2} \leq C\left\{\int_{0}^{T}\left(z_{x}^{2}(0, t)+w_{x}^{2}(0, t)\right) d t\right. \\
\left.+\int_{0}^{T}\|V\|_{\left[L^{2}(\omega)\right]^{2}}^{2} d t+\left\|z_{0}\right\|_{H^{-3}(0, L)}^{2}+\left\|w_{0}\right\|_{H^{-3}(0, L)}^{2}\right\}
\end{aligned}
$$

for any solution of (1.1)-(1.3).

We argue by contradiction. Suppose that (2.50) does not hold. Then, there exists a sequence of functions $V_{n}=\left(\begin{array}{c}z_{n} \\ w_{n}\end{array}\right) \in L^{\infty}(0, T ; X) \cap L^{2}\left(0, T ;\left[H_{0}^{1}(0, L)\right]^{2}\right)$ solving

$$
\left\{\begin{array}{l}
V_{n, t}+V_{n, x}+D V_{n, x x x}+\lambda H\left(V_{n}\right) U_{x}+\lambda H(U) V_{n, x}+\mathcal{L}(x, B) V_{n}=0, \text { in }(0, L) \times(0, T) \\
V_{n}(0, t)=V_{n}(L, t)=V_{n, x}(L, t)=0, \quad t \in(0, T) \\
V_{n}(x, 0)=V_{n, 0}(x)=\left(\begin{array}{c}
z_{n}(x, 0) \\
w_{n}(x, 0)
\end{array}\right)=\left(\begin{array}{c}
z_{n, 0}(x) \\
w_{n, 0}(x)
\end{array}\right), \quad 0<x<L,
\end{array}\right.
$$

satisfying

$$
\left\|V_{n, 0}\right\|_{X}^{2}=\left\|z_{n, 0}\right\|_{L^{2}(0, L)}^{2}+\left\|w_{n, 0}\right\|_{L^{2}(0, L)}^{2} \leq R^{2}, \quad \forall n \in \mathbb{N}
$$

and such that

$$
\frac{\left\|V_{n}\right\|_{L^{4}\left(0, T ;\left[L^{4}(0, L)\right]^{2}\right)}^{2}}{\int_{0}^{T}\left(z_{n, x}^{2}(0, t)+w_{n, x}^{2}(0, t)\right) d t+\int_{0}^{T}\left\|V_{n}\right\|_{Y(\omega)}^{2} d t+\left\|V_{n, 0}\right\|_{\left[H^{-3}(0, L)\right]^{2}}^{2}}
$$

goes to infinity as $n \rightarrow \infty$. Let

$$
\sigma_{n}=\left(\left\|z_{n}\right\|_{L^{4}\left(0, T ; L^{4}(0, L)\right)}^{2}+\left\|w_{n}\right\|_{L^{4}\left(0, T ; L^{4}(0, L)\right)}^{2}\right)^{\frac{1}{2}}
$$

and consider

$$
\Phi_{n}(x, t)=\left(\begin{array}{c}
\phi_{n}(x, t) \\
\psi_{n}(x, t)
\end{array}\right)=\frac{1}{\sigma_{n}}\left(\begin{array}{c}
z_{n}(x, t) \\
w_{n}(x, t)
\end{array}\right), \quad n \in \mathbb{N} .
$$


For each $n \in \mathbb{N}$ the function $\Phi_{n}$ solves

$$
\left\{\begin{array}{l}
\Phi_{n, t}+\Phi_{n, x}+D \Phi_{n, x x x}+\lambda H\left(\Phi_{n}\right) U_{x}+\lambda H(U) \Phi_{n, x}+\mathcal{L}(x, B) \Phi_{n}=0, \\
\Phi_{n}(0, t)=\Phi_{n}(L, t)=\Phi_{n, x}(L, t)=0 \\
\Phi_{n}(x, 0)=\Phi_{n, 0}(x)=\left(\begin{array}{c}
\phi_{n}(x, 0) \\
\psi_{n}(x, 0)
\end{array}\right)=\left(\begin{array}{c}
\phi_{n, 0}(x) \\
\psi_{n, 0}(x)
\end{array}\right)=\left(\begin{array}{c}
\frac{w_{n}(x, 0)}{\sigma_{n}} \\
\frac{w_{n}(x, 0)}{\sigma_{n}}
\end{array}\right),
\end{array}\right.
$$

in $(0, L) \times(0, T)$, and satisfies

$$
\left\|\phi_{n}\right\|_{L^{4}\left(0, T ; L^{4}(0, L)\right)}^{2}+\left\|\psi_{n}\right\|_{L^{4}\left(0, T ; L^{4}(0, L)\right)}^{2}=1
$$

and

$$
\int_{0}^{T}\left(\phi_{n, x}^{2}(0, t)+\psi_{n, x}^{2}(0, t)\right) d t+\int_{0}^{T}\left\|\Phi_{n}\right\|_{Y(\omega)}^{2} d t+\left\|\Phi_{n, 0}\right\|_{\left[H^{-3}(0, L)\right]^{2}}^{2} \rightarrow 0,
$$

as $n \rightarrow \infty$. Moreover,

a) $\left\{\Phi_{n, 0}\right\}_{n \in \mathbb{N}}$ is bounded in $X$;

b) $\left\{\sigma_{n}\right\}_{n \in \mathbb{N}}$ is bounded in $\mathbb{R}$;

c) $\left\{\Phi_{n}\right\}_{n \in \mathbb{N}}$ is bounded in $L^{\infty}(0, T ; X) \cap L^{2}\left(0, T ;\left[H_{0}^{1}(0, L)\right]^{2}\right)$;

d) $\left\{H\left(\Phi_{n}\right) U_{x}\right\}_{n \in \mathbb{N}}$ and $\left\{H(U) \Phi_{n, x}\right\}_{n \in \mathbb{N}}$ are bounded in

e) $\left\{\Phi_{n, t}\right\}_{n \in \mathbb{N}}$ is bounded in $L^{2}\left(0, T ;\left[H^{-2}(0, L)\right]^{2}\right)$;

$f)$ there exists $s>0$ such that $\left\{\Phi_{n}\right\}_{n \in \mathbb{N}}$ is bounded in

$L^{4}\left(0, T ;\left[H^{s}(0, L)\right]^{2}\right)$, the embedding $H^{s}(0, L) \hookrightarrow L^{4}(0, L)$ being compact.

Indeed,

a) Multiplying inequality (2.49) by $\frac{1}{\sigma_{n}^{2}}$ and using (2.55) we have

$$
\begin{gathered}
\int_{0}^{L}\left(\phi_{n}^{2}(x, 0)+\psi_{n}^{2}(x, 0)\right) d x \leq \underbrace{c\left(\left\|\phi_{n}\right\|_{L^{4}\left(0, T ; L^{4}(0, L)\right)}^{2}+\left\|\psi_{n}\right\|_{L^{4}\left(0, T ; L^{4}(0, L)\right)}^{2}\right)}_{\text {equal to one by (2.57) }} \\
+\underbrace{\left(1+a_{3}\right) \int_{0}^{T}\left(\phi_{n, x}^{2}(0, t)+\psi_{n, x}^{2}(0, t)\right) d t+2 \int_{0}^{T}\left\|\Phi_{n}\right\|_{Y(\omega)}^{2} d t .}_{\text {which is bounded by (2.58) }}
\end{gathered}
$$

Thus,

$$
\left\|\Phi_{n, 0}\right\|_{X}^{2}=\left\|\phi_{n}(\cdot, 0)\right\|_{L^{2}(0, L)}^{2}+\left\|\psi_{n}(\cdot, 0)\right\|_{L^{2}(0, L)}^{2} \leq C, \quad \forall n \in \mathbb{N},
$$

for some constant $C>0$.

b) Letting $t=0$ in (2.55), the boundedness for $\left\{\sigma_{n}\right\}_{n \in \mathbb{N}}$ follows from (2.52) and (2.58).

c) Taking (2.59) and (2.60) into account, we can proceed as in Step 4 of Lemma 2.4 (see (2.42) and (2.43) ). 
d) Applying the Young and Hölder inequalities we get

$$
\begin{aligned}
& \left\|\left(u \phi_{n}\right)_{x}\right\|_{L^{2}\left(0, T ; L^{1}(0, L)\right)}=\left(\int_{0}^{T}\left\|\left(u \phi_{n}\right)_{x}\right\|_{L^{1}(0, L)}^{2} d t\right)^{\frac{1}{2}} \\
& \leq\left(\int_{0}^{T}\left(\|u\|_{H_{0}^{1}(0, L)}\left\|\phi_{n}\right\|_{L^{2}(0, L)}+\|u\|_{L^{2}(0, L)}\left\|\phi_{n}\right\|_{H_{0}^{1}(0, L)}\right)^{2} d t\right)^{\frac{1}{2}} \\
& \leq\left(2 \int_{0}^{T}\left(\|u\|_{H_{0}^{1}(0, L)}^{2}\left\|\phi_{n}\right\|_{L^{2}(0, L)}^{2}+\|u\|_{L^{2}(0, L)}^{2}\left\|\phi_{n}\right\|_{H_{0}^{1}(0, L)}^{2}\right) d t\right)^{\frac{1}{2}} \\
& \leq\left(2\left\|\phi_{n}\right\|_{L^{\infty}\left(0, T ; L^{2}(0, L)\right)}^{2}\|u\|_{L^{2}\left(0, T ; H_{0}^{1}(0, L)\right)}^{2}+2\|u\|_{L^{\infty}\left(0, T ; L^{2}(0, L)\right)}^{2}\left\|\phi_{n}\right\|_{L^{2}\left(0, T ; H_{0}^{1}(0, L)\right)}^{2}\right)^{\frac{1}{2}} \\
& \leq \sqrt{2}\left(\left\|\phi_{n}\right\|_{L^{\infty}\left(0, T ; L^{2}(0, L)\right)}\|u\|_{L^{2}\left(0, T ; H_{0}^{1}(0, L)\right)}+\|u\|_{L^{\infty}\left(0, T ; L^{2}(0, L)\right)}\left\|\phi_{n}\right\|_{L^{2}\left(0, T ; H_{0}^{1}(0, L)\right)}\right) .
\end{aligned}
$$

Combining Theorem 2.1 and (2.61) we deduce that

$$
\left\|\left(u \phi_{n}\right)_{x}\right\|_{L^{2}\left(0, T ; L^{1}(0, L)\right)} \leq C
$$

for some constant $C>0$. Analogously, we bound the other terms in (2.62).

e) Taking the results above into account, it is sufficient to observe that

$$
\Phi_{n, t}=-\Phi_{n, x}-\Phi_{n, x x x}-\lambda H\left(\Phi_{n}\right) U-\lambda H(U) \Phi_{n}-\mathcal{L}(x, B) \Phi_{n} .
$$

f) In fact, since $\left\{\Phi_{n}\right\}_{n \in \mathbb{N}}$ is bounded in $L^{2}\left(0, T ;\left[H_{0}^{1}(0, L)\right]^{2}\right) \cap L^{\infty}(0, T ; X)$, by interpolation we can deduce that $\left\{\phi_{n}\right\}_{n \in \mathbb{N}}$ and $\left\{\psi_{n}\right\}_{n \in \mathbb{N}}$ are bounded in

$$
\left[L^{2}\left(0, T ; H_{0}^{1}(0, L)\right), L^{q}\left(0, T ; L^{2}(0, L)\right)\right]_{\theta}=L^{4}\left(0, T ;\left[H_{0}^{1}(0, L), L^{2}(0, L)\right]_{\theta}\right),
$$

where $\frac{1}{4}=\frac{1-\theta}{2}+\frac{\theta}{q}$ and $0<\theta<1$. On the other hand, according to [12],

$$
\left[H_{0}^{1}(0, L), L^{2}(0, L)\right]_{\theta}=H_{0}^{s}(0, L) \quad \text { if } s=1-\theta \neq \frac{1}{2},
$$

and due to the Rellich-Kondrachov theorem we obtain that

$$
H^{s}(0, L) \hookrightarrow L^{4}(0, L) \quad \text { if } \frac{1}{4}>\frac{1}{2}-\frac{s}{1} .
$$

Thus, $s>\frac{1}{4}$, and, consequently, $\theta<\frac{3}{4}$. Choosing $q=8$ and $\theta=\frac{2}{3}$, the claim holds with $s=\frac{1}{3}$ :

$$
\left[H_{0}^{1}(0, L), L^{2}(0, L)\right]_{\frac{2}{3}}=H_{0}^{\frac{1}{3}}(0, L)=H^{\frac{1}{3}}(0, L) .
$$

Furthermore, the embedding $H^{\frac{1}{3}}(0, L) \hookrightarrow L^{4}(0, L)$ is compact.

The statement above allows us to conclude that $\left\{\phi_{n}\right\}_{n \in \mathbb{N}}$ and $\left\{\psi_{n}\right\}_{n \in \mathbb{N}}$ belong to the set

$$
\mathcal{W}=\left\{w \in L^{4}\left(0, T ; H^{\frac{1}{3}}(0, L)\right) ; \frac{d w}{d t} \in L^{2}\left(0, T ; H^{-2}(0, L)\right)\right\} .
$$

Then, by classical compactness results [24], we can extract a subsequence of $\left\{\Phi_{n}\right\}_{n \in \mathbb{N}}$, still denoted by same index $n$, such that

$$
\Phi_{n} \longrightarrow \Phi:=\left(\begin{array}{c}
\phi \\
\psi
\end{array}\right) \quad \text { strongly in } L^{4}\left(0, T ;\left[L^{4}(0, L)\right]^{2}\right), \quad \text { as } n \rightarrow \infty .
$$


Consequently, by (2.57),

$$
\|\phi\|_{L^{4}\left(0, T ; L^{4}(0, L)\right)}^{2}+\|\psi\|_{L^{4}\left(0, T ; L^{4}(0, L)\right)}^{2}=1 .
$$

Moreover, using lower semicontinuity, we obtain that

$$
\begin{aligned}
& 0=\liminf _{n \rightarrow \infty}\left\{\int_{0}^{T}\left(\phi_{n, x}^{2}(0, t)+\psi_{n, x}^{2}(0, t)\right) d t+\int_{0}^{T}\left\|\Phi_{n}\right\|_{Y(\omega)}^{2} d t\right. \\
& \left.+\left\|\phi_{n, 0}\right\|_{H^{-3}(0, L)}^{2}+\left\|\psi_{n, 0}\right\|_{H^{-3}(0, L)}^{2}\right\} \\
& \geq \int_{0}^{T}\left(\phi_{x}^{2}(0, t)+\psi_{x}^{2}(0, t)\right) d t+\int_{0}^{T}\|\Phi\|_{Y(\omega)}^{2} d t+\left\|\phi_{0}\right\|_{H^{-3}(0, L)}^{2}+\left\|\psi_{0}\right\|_{H^{-3}(0, L)}^{2},
\end{aligned}
$$

which, in particular, implies that

$$
\phi(x, 0)=\psi(x, 0)=0 .
$$

Thus, the limit $\Phi$ which solves the system

$$
\left\{\begin{array}{l}
\Phi_{t}+\Phi_{x}+D \Phi_{x x x}+\lambda H(\Phi) U_{x}+\lambda H(U) \Phi_{x}+\mathcal{L}(x, B) \Phi=0, \quad \text { in }(0, L) \times(0, T) \\
\Phi(0, t)=\Phi(L, t)=\Phi_{x}(L, t)=0, \quad t \in(0, T) \\
\Phi(x, 0)=\Phi_{0}(x)=0, \quad 0<x<L
\end{array}\right.
$$

is identically zero, i.e., $\phi \equiv \psi \equiv 0$. This contradicts (2.65) and, necessarily, (2.50) has to be valid. This completes the proof of Lemma 2.5.

We are now in condition to prove Theorem 2.2.

Proof. Proof of Theorem 2.2:

Let $\left(\begin{array}{c}u_{0} \\ v_{0}\end{array}\right) \in X$. Differentiating system (1.1)-(1.3) with respect to $t$, we obtain the system (2.2)-(2.4) with

$$
V(x, 0)=V_{0}(x)=\left(\begin{array}{c}
z_{0}(x) \\
w_{0}(x)
\end{array}\right)=\left(\begin{array}{c}
u_{t}(x, 0) \\
w_{t}(x, 0)
\end{array}\right) \in\left[H^{-3}(0, L)\right]^{2} .
$$

On the other hand, if $U_{x}(0, t)=0$ and $U=0$ in $\omega \times(0, T)$, then $V_{x}(0, t)=0$ and $V$ vanishes in $\omega \times(0, T)$ as well. Consequently, by assumption on the damping $\mathcal{L}(x, B)$, we conclude that $V \equiv 0$ in $\omega \times(0, T)$ and according to Lemma 2.5, we obtain that $V_{0} \in X$.

Now, combining Lemma 2.4 and system (1.1), we get

$$
U_{t}=\left(\begin{array}{c}
u_{t} \\
v_{t}
\end{array}\right)=\left(\begin{array}{c}
z \\
w
\end{array}\right)=V \in L^{2}\left(0, T ;\left[H_{0}^{1}(0, L)\right]^{2}\right) \cap L^{\infty}(0, T ; X)
$$

and

$$
D U_{x x x}=-U_{t}-U_{x}-\lambda H(U) U_{x}-\mathcal{L}(x, B) U, \quad \text { in } \Omega \times(0, \infty) .
$$

Consequently, by (2.67), (2.68) and Theorem 2.1 it follows that

$$
U \in L^{2}\left(0, T ;\left[H^{3}(0, L)\right]^{2}\right) \cap H^{1}(0, T ; X) .
$$

Now, using the UCP proved in [6] (Corollary 3.5) we have $U \equiv 0$. 


\section{Proof of the main result.}

Proof. We will use methods as in [16. We first take the inner product in $X$ of (1.1) with $\left(\begin{array}{c}(T-t) u \\ (T-t) v\end{array}\right)$ and integrating over $(0, L) \times(0, T)$ to obtain the identity

$$
\begin{aligned}
T \int_{0}^{L}\left(u_{0}^{2}+v_{0}^{2}\right) d x= & \int_{0}^{T} \int_{0}^{L}\left(u^{2}+v^{2}\right) d x d t+\int_{0}^{T}(T-t)\left(u_{x}^{2}(0, t)+v_{x}^{2}(0, t)\right) d t \\
& +2 a_{3} \int_{0}^{T}(T-t) u_{x}(0, t) v_{x}(0, t) d t+2 \int_{0}^{T}(\mathcal{L}(x, B) U, U) d t .
\end{aligned}
$$

Consequently,

$$
\begin{aligned}
\int_{0}^{L}\left(u_{0}^{2}+v_{0}^{2}\right) d x \leq & \frac{1}{T} \int_{0}^{T} \int_{0}^{L}\left(u^{2}+v^{2}\right) d x d t+\left(1+a_{3}\right) \int_{0}^{T}\left(u_{x}^{2}(0, t)+v_{x}^{2}(0, t)\right) d t \\
& +2 \int_{0}^{T}\|U\|_{Y(\omega)}^{2} d t .
\end{aligned}
$$

Now, we claim that, for any $T>0$ and $R>0$, there exists a constant $C=C(R, T)>0$ such that

$$
\int_{0}^{T} \int_{0}^{L}\left(u^{2}+v^{2}\right) d x d t \leq C\left[\int_{0}^{T}\left(u_{x}^{2}(0, t)+v_{x}^{2}(0, t)\right) d t+\int_{0}^{T}\|U\|_{Y(\omega)}^{2} d t\right]
$$

for any solution $U$ of (1.1)-(1.3), whenever $\left\|U_{0}\right\|_{X}^{2} \leq R^{2}$.

We argue by contradiction. Suppose that (3.3) is not true. Then, there exists a sequence of functions $\left\{U_{n}\right\}_{n \in \mathbb{N}} \in L^{\infty}(0, T ; X) \cap L^{2}\left(0, T ;\left[H_{0}^{1}(0, L)\right]^{2}\right)$ that is a solution of

$$
\left\{\begin{array}{l}
U_{n, t}+U_{n, x}+D U_{n, x x x}+H\left(U_{n}\right) U_{n, x}+\mathcal{L}(x, B) U_{n}=0, \quad x \in(0, L), t>0, \\
U_{n}(0, t)=U_{n}(L, t)=U_{n, x}(L, t)=0, \quad t>0, \\
U_{n}(x, 0)=U_{n, 0}(x), \quad 0<x<L .
\end{array}\right.
$$

Moreover,

$$
\left\|U_{n, 0}\right\|_{X}^{2} \leq R^{2}, \quad \forall n \in \mathbb{N}
$$

and

$$
\lim _{n \rightarrow \infty} \frac{\left\|u_{n}\right\|_{L^{2}\left(0, T ; L^{2}(0, L)\right)}^{2}+\left\|v_{n}\right\|_{L^{2}\left(0, T ; L^{2}(0, L)\right)}^{2}}{\int_{0}^{T}\left(u_{n, x}^{2}(0, t)+v_{n, x}^{2}(0, t)\right) d t+\int_{0}^{T}\|U\|_{Y(\omega)}^{2} d t}=\infty
$$

Let

$$
\sigma_{n}=\left(\left\|u_{n}\right\|_{L^{2}\left(0, T ; L^{2}(0, L)\right)}^{2}+\left\|v_{n}\right\|_{L^{2}\left(0, T ; L^{2}(0, L)\right)}^{2}\right)^{\frac{1}{2}}=\left\|U_{n}\right\|_{X}
$$

and consider

$$
Z_{n}(x, t)=\frac{1}{\sigma_{n}} U_{n}(x, t)=\frac{1}{\sigma_{n}}\left(\begin{array}{c}
u_{n}(x, t) \\
v_{n}(x, t)
\end{array}\right) .
$$


For each $n \in \mathbb{N}, Z_{n}$ satisfies

$$
\left\{\begin{array}{l}
Z_{n, t}+Z_{n, x}+D Z_{n, x x x}+\sigma_{n} H\left(Z_{n}\right) Z_{n, x}+a(x) Z_{n}=0, \quad x \in(0, L), t>0 \\
Z_{n}(0, t)=Z_{n}(L, t)=Z_{n, x}(L, t)=0, \quad t>0 \\
Z_{n}(x, 0)=Z_{n, 0}=\frac{U_{n, 0}}{\sigma_{n}}, \quad 0<x<L, \\
\left\|Z_{n}\right\|_{L^{2}(0, T ; X)}^{2}=1
\end{array}\right.
$$

and

$$
\lim _{n \rightarrow \infty} \int_{0}^{T}\left(u_{n, x}^{2}(0, t)+v_{n, x}^{2}(0, t)\right) d t+\int_{0}^{T}\left\|Z_{n}\right\|_{Y(\omega)}^{2} d t=0 .
$$

Following the arguments used in the proof of Lemma 2.5, we can prove that

$$
Z_{n} \rightarrow Z \text { strongly in } L^{2}(0, T ; X), \text { as } n \rightarrow \infty,
$$

where the limit function $Z$ solves

$$
\left\{\begin{array}{l}
Z_{t}+Z_{x}+D Z_{x x x}+H(Z) Z_{x}+a(x) Z=0, \quad x \in(0, L), t \in(0, T) \\
Z(0, t)=Z(L, t)=Z_{x}(L, t)=0, \quad t \in(0, T) .
\end{array}\right.
$$

In addition,

$$
\left\{\begin{array}{l}
Z_{x}(0, t)=0, \quad t \in(0, T) \\
Z \equiv 0, \quad \text { in } \omega \times(0, T) .
\end{array}\right.
$$

Consequently, from Theorem 2.2, we conclude that $Z \equiv 0$ in $(0, L) \times(0, T)$, which contradicts (3.10). Indeed, since $Z_{n} \rightarrow Z$ strongly in $L^{2}(0, T ; X)$, from (3.10) we deduce that $\|Z\|_{L^{2}(0, T ; X)}^{2}=1$. Thus,

$$
\int_{0}^{L}\left(u_{0}^{2}+v_{0}^{2}\right) d x \leq C\left[\int_{0}^{T}\left(u_{x}^{2}(0, t)+v_{x}^{2}(0, t)\right) d t+\int_{0}^{T}\|U\|_{Y(\omega)}^{2} d t\right]
$$

for some $C=C(R, T)$. On the other hand, from (1.7) and (1.9) we have

$$
\begin{aligned}
\int_{0}^{T}\left[u^{2}(x, T)+v^{2}(x, T)\right] d x d t \leq & \int_{0}^{L}\left(u_{0}^{2}+v_{0}^{2}\right) d x-2 \int_{0}^{T}\|U\|_{Y(\omega)}^{2} d t \\
& +\left(1-a_{3}\right) \int_{0}^{T}\left(u_{x}^{2}(0, t)+v_{x}^{2}(0, t)\right) d t .
\end{aligned}
$$

Inequalities (3.15) and (3.16) give the exponential decay of the energy $E(t)$. Indeed, let $\kappa=\frac{C\left(1+a_{3}\right)}{1-a_{3}}$, where $C$ is the constant that appears in (3.15). Then,

$$
\begin{aligned}
& (1+\kappa)\left(\|u(\cdot, T)\|_{L^{2}(0, L)}^{2}+\|v(\cdot, T)\|_{L^{2}(0, L)}^{2}\right) \\
& \quad \leq \kappa\left(\left\|u_{0}\right\|_{L^{2}(0, L)}^{2}+\left\|v_{0}\right\|_{L^{2}(0, L)}^{2}\right)-2(\kappa-C) \int_{0}^{T}\|U\|_{Y(\omega)}^{2} d t \\
& \quad-\left(1-a_{3}\right) \int_{0}^{T}\left(u_{x}^{2}(0, t)+v_{x}^{2}(0, t)\right) d t \leq \kappa\left(\left\|u_{0}\right\|_{L^{2}(0, L)}^{2}+\left\|v_{0}\right\|_{L^{2}(0, L)}^{2}\right) .
\end{aligned}
$$

Recalling that $0<a_{3}<1$, we have

$$
E(T) \leq \gamma E(0)
$$


where $0<\gamma<1$. The semigroup property implies the conclusion of Theorem 1.1 .

\section{REFERENCES}

[1] E. Bisognin, V. Bisognin and G.P. Menzala, Exponential stabilization of a coupled system of Korteweg-de Vries equations with localized damping, Adv. Diff. Eq., 8 (2003), 443-469. MR.1972596 (2004c:35349)

[2] J. Bona, G. Ponce, J.C. Saut and M.M. Tom, A model system for strong interaction between internal solitary waves, Comm. Math. Phys., 143 (1992), 287-313. MR1145797 (93e:35086)

[3] E. Cerpa, Exact controllability of a nonlinear Korteweg-de Vries equation on a critical spatial domain, SIAM J. Control Optim., 46 (2007), 877-899. MR2338431 (2008f:93008)

[4] M. Chapouly, Global controllability of a nonlinear Korteweg-de Vries equation, Commun. Contemp. Math.11 (2009), 495-521. MR.2538210 (2010j:93008)

[5] J. Coron and E. Crépeau, Exact boundary controllability of a nonlinear KdV equation with critical lengths, J. Eur. Math. Soc. (JEMS)6 (2004), 367-398. MR2060480(2005b:93016)

[6] M. Davila, On the unique continuation property for a coupled system of Korteweg-de Vries equations, Ph.D. Thesis, Institute of Mathematics, Federal University of Rio de Janeiro, Brazil, 1994.

[7] J.A. Gear and R. Grimshaw, Weak and strong interaction between internal solitary waves, Stud. in Appl. Math. 70 (1984), 235-258. MR742590 (85i:76013)

[8] T. Kato, On the Cauchy problem for the (generalized) Korteweg-de Vries equation, Stud. Appl. Math. Adv., in Math. Suppl. Stud. 8 (1983), 93-128. MR759907 (86f:35160)

[9] F. Linares and M. Panthee, On the Cauchy problem for a coupled system of KdV equations, Commun. Pure Appl. Anal. 3 (2004), 417-431. MR2098292 (2005h:35308)

[10] F. Linares and A. F. Pazoto, On the exponential decay of the critical generalized Korteweg-de Vries equation with localized damping, Proc. Amer. Math. Soc. 135 (2007), 1515-1522. MR2276662 (2008e:35168)

[11] J. Lions, Contrôlabilité exacte, perturbations et stabilisation de systèmes distribués, Tome 1, Contrôlabilité exacte, Collection de Recherches en Mathématiques Appliquées, 8, Masson, Paris (1988). MR963060 (89k:93017)

[12] J. Lions and E. Magenes, Problèmes aux limites non homogènes et applications, vol. 1, Dunod, Paris (1968). MR0247243(40:512)

[13] C. P. Massarolo and A. F. Pazoto, Uniform stabilization of a nonlinear coupled system of Kortewegde Vries equations as a singular limit of the Kuramoto-Sivashinsky system, Diff. Int. Eq. 22 (2009), 53-68. MR2483012(2010h:35349)

[14] C. P. Massarolo, G. P. Menzala and A. F. Pazoto, On the uniform decay for the Korteweg-de Vries equation with weak damping, Math. Methods Appl. Sci. 30 (2007), 1419-1435. MR2337386 (2008c:35282)

[15] G.P. Menzala and E. Zuazua, Decay rates for the von Kármán system of thermoelastic plates, Diff. Int. Eq. 11 (1998), 755-770. MR1666187 (99m:35244)

[16] G.P. Menzala, C.F. Vasconcellos and E. Zuazua, Stabilization of the Korteweg-de Vries equation with localized damping, Quarterly of Appl. Math. LX (2002), 111-129. MR1878262 (2002j:35273)

[17] S. Micu and J.H. Ortega, On the controllability of a linear coupled system of Korteweg-de Vries equations, Mathematical and numerical aspects of wave propagation (Santiago de Compostela, 2000), SIAM, Philadelphia, PA, 2000, 1020-1024. MR1786022

[18] S. Micu, J.H. Ortega and A. F. Pazoto, On the controllability of a nonlinear coupled system of Korteweg-de Vries equations, Commun. Contemp. Math. 11 (2009), 799-827. MR2561938

[19] A. F. Pazoto, Unique continuation and decay for the Korteweg-de Vries equation with localized damping, ESAIM Control Optimization and Calculus of Variations 11 (2005), 473-486. MR2148854 (2006b:35292)

[20] L. Rosier and B.-Y. Zhang, Global stabilization of the generalized Korteweg-de Vries equation posed on a finite domain, SIAM J. Control Optim. 45 (2006), 927-956. MR2247720(2007h:35297)

[21] L. Rosier, Control of the surface of a fluid by a wavemaker, ESAIM Control Optimization and Calculus of Variations 10 (2004), 346-380. MR2084328 (2005h:93091)

[22] L. Rosier, Exact boundary controllability for the Korteweg-de Vries equation on a bounded domain, ESAIM Control Optimization and Calculus of Variations 2 (1997), 33-55. MR1440078 (98d:93016)

[23] J.C. Saut and B. Scheurer, Unique continuation for some evolution equations, J. Diff. Equations 66 (1987), 118-139. MR871574 (88a:35115) 
[24] J. Simon, Compact sets in the space $L^{p}(0, T ; B)$, Annali di Matematica Pura ed Applicata CXLVI (1987), 65-96. MR916688 (89c:46055)

[25] F. Trêves, Linear Partial Differential Equations with constant coefficients, Gordon and Breach, New York/London/Paris (1966). MR0224958 (37:557)

[26] O. P. Vera Villagran, Gain of regularity of the solutions of a coupled system of equations of Korteweg-de Vries type, Ph.D. Thesis, Institute of Mathematics, Federal University of Rio de Janeiro, Brazil, 2001.

[27] B.Y. Zhang, Unique continuation for the Korteweg-de Vries equation, SIAM J. Math. Anal. 23 (1992), 55-71. MR1145162 (92k:35252)

[28] B.Y. Zhang, Exact boundary controllability of the Korteweg-de Vries equation, SIAM J. Control Opt. 37 (1999), 543-565. MR1670653 (2000b:93010)

[29] E. Zuazua, Exponential decay for the semilinear wave equation with locally distributed damping, Comm. Partial Diff. Eq. 15 (1990), 205-235. MR.1032629 (91b:35076) 